\title{
Revisão do kit básico de medicamentos para cirurgias de descompressão do túnel do carpo: proposta para melhorar a economia e a segurança do paciente
}

\author{
Review of the basic drug kit for carpal tunnel decompression surgeries: proposal to improve \\ patient economy and safety
}

Revisión del kit básico de medicamentos para las cirugías de descompresión del túnel carpiano: propuesta para mejorar la economía y la seguridad del paciente

\section{Resumo}

Objetivo: propor a adoção de um kit de medicamentos específico para procedimentos cirúrgicos de descompressão do túnel do carpo, para substituir o kit farmacoterapêutico básico atualmente utilizado. Métodos: trata-se de projeto de desenvolvimento experimental envolvendo observação direta, levantamento de dados administrativos, estudo de evidências científicas e comparação econômica para propor o novo kit. Resultados: a observação direta indicou que os procedimentos adotados por vezes divergiam daquelas baseadas em evidências científicas; o perfil de utilização de medicamentos demonstrou que o kit atual é subutilizado e favorece o uso irracional de medicamentos; conhecendo-se o perfil de uso e as evidências científicas, foi possível propor um novo kit mais econômico, que permite mitigar o uso irracional e contribui para as estratégias de segurança do paciente. Conclusão: a adoção do novo kit proposto pode trazer benefícios à instituição tanto pela economia quanto pela contribuição para melhores resultados assistenciais e maior segurança do paciente.

Palavras-chave: Segurança do paciente; Sistemas de Distribuição de Medicamentos em Hospital; Serviço de Farmácia Hospitalar; Gastos em Saúde; Farmacoeconomia.

\begin{abstract}
Objective: to propose the adoption of a specific drug kit for surgical procedures for decompression of the carpal tunnel, to replace the basic pharmacotherapeutic kit currently used. Methods: this is an experimental development project involving direct observation, administrative data collection, study of scientific evidence and economic comparison to propose the new kit. Results: direct observation indicated that the procedures adopted sometimes diverged from those based on scientific evidence; the profile of medication use showed that the current kit is underused and favors the irrational use of medications; knowing the usage profile and the scientific evidence, it was possible to propose a new, more economical kit, which allows to mitigate irrational use and contributes to patient safety strategies. Conclusion: the adoption of the proposed new kit can bring benefits to the institution both in terms of economy and contribution to better care outcomes and greater patient safety.
\end{abstract}

Keywords: Patient safety; Medication Systems, Hospital; Pharmacy Service, Hospital; Health Expenditures; Economics, Pharmaceutical.

\section{Resumen}

Objetivo: proponer la adopción de un kit de fármacos específico para procedimientos quirúrgicos de descompresión del túnel carpiano, que pueda sustituir a lo kit farmacoterapéutico básico utilizado actualmente. Métodos: se trata de un proyecto de desarrollo experimental que implica observación directa, recopilación de datos administrativos, estudio de evidencia científica y comparación económica para proponer el nuevo kit. Resultados: la observación directa indicó que los procedimientos adoptados en ocasiones divergieron de los basados en evidencia científica; el perfil de uso de medicamentos mostró que el kit actual está infrautilizado y favorece el uso irracional de medicamentos; 
conociendo el perfil de uso y la evidencia científica, fue posible proponer un nuevo kit más económico, que permite mitigar el uso irracional y contribuye a las estrategias de seguridad del paciente. Conclusión: la adopción del nuevo kit propuesto puede traer beneficios a la institución tanto en términos de economía como de contribución a mejores resultados de atención y mayor seguridad del paciente.

Palabras clave: Seguridad del paciente; Sistemas de Medicación en Hospital; Servicio de Farmacia en Hospital; Gastos en Salud; Economía Farmacéutica.

\section{Introdução}

Use o parágrafo como modelo Estabelecimentos prestadores de serviços de saúde têm por finalidade a promoção, a proteção, a recuperação e a reabilitação da saúde do indivíduo ou a prevenção da doença. Diversos procedimentos são realizados na tentativa de alcançar esses objetivos. A execução destes processos está diretamente relacionada à possibilidade da ocorrência de erros. Algumas destas falhas influenciam diretamente na segurança do paciente (Paraná, 2001).

De maneira geral, a segurança do paciente tem sido centro de diversas discussões nos últimos anos. O debate vem se ampliando desde a publicação de To Err Is Human: Building a Safer Health System pelo Institute of Medicine (IOM), que constatou que erros médicos matam entre 44.000 a 98.000 pessoas nos EUA todos os anos. Estes dados demonstram que erros médicos matam mais pessoas, isoladamente, do que AIDS, acidentes rodoviários e câncer de mama, segundo dados referentes ao mesmo país (Kohn et al., 1999).

Muitos são os tipos de erros que comprometem a segurança do paciente. Dentre eles, podem-se mencionar os de medicação. Este tipo de erro tem a falta de rastreabilidade dos medicamentos como uma de suas possíveis causas (brasil, 2009).

Ao rastrear um medicamento, é possível acompanhar desde a sua produção até o seu uso. Assim, é viável identificar problemas relacionados a lotes específicos e/ou problemas que não estejam diretamente ligados à produção do medicamento em si, tais como falhas no armazenamento, na manipulação, na dispensação, na administração e no uso, reforçando ainda mais a relação entre rastreabilidade e erro de medicação (Lei n $\left.{ }^{\circ} 13.410,2016\right)$.

Devido à importância do tema, em 2009, o Governo Federal criou o Sistema Nacional de Controle de Medicamentos (SNCM), através da Lei $\mathrm{N}^{\circ} 11.903$, alterada posteriormente, pela Lei $\mathrm{n}^{\circ}$ 13.410/2016, "visando a controlar a produção, a distribuição, a comercialização, a dispensação e a prescrição médica, odontológica e, caso contenha medicamento de uso humano, veterinária, assim como os demais tipos de movimentação previstos pelos controles sanitários" (Lei n 11.903, 2009; Lei $\left.n^{\circ} 13.410,2016\right)$.

A rastreabilidade auxilia em uma gestão mais eficiente destes insumos. Além do controle da segurança e da eficácia dos medicamentos utilizados, é possível que se façam previsões relacionadas ao seu consumo. Desta forma, as competências gerenciais do profissional farmacêutico também são aproveitadas, gerando benefícios não somente para os pacientes, mas também para a instituição de saúde, no que tange o aspecto financeiro da mesma (Míriam Elias Cavallini \& Marcelo Polacow Bisson, 2002).

Com base no racional exposto, este projeto vem propor a adoção de um kit de medicamentos específico contendo medicamentos para procedimentos cirúrgicos de descompressão do túnel do carpo, para substituir o kit farmacoterapêutico básico atualmente utilizado, que contém medicamentos para todas as cirurgias.

\section{Metodologia}

Trata-se de um projeto de desenvolvimento experimental (CNPq, 2021), desenvolvido em cinco etapas que envolveram pesquisa na literatura, observação in loco da utilização de medicamentos em procedimentos cirúrgicos, levantamento de dados administrativos para acompanhamento do uso de medicamentos e a proposta de um novo kit específico 
para o procedimento cirúrgico eleito para o estudo.

A primeira etapa do projeto consistiu em realizar uma busca por protocolos de centros de referência disponíveis na literatura para cirurgias de descompressão do túnel do carpo, visando maior compreensão do tema. Os protocolos encontrados entre novembro de 2018 e junho de 2019 foram analisados com vistas aos medicamentos elegíveis em cada caso.

$\mathrm{Na}$ segunda etapa, os procedimentos cirúrgicos de microneurólises foram acompanhados presencialmente por um período de 4 dias, observando-se especificamente a utilização dos itens constantes no kit básico já em uso, a fim de comparar o real uso com as recomendações verificadas na literatura especializada.

Microneurólises são procedimentos simples e, na instituição onde o estudo foi realizado, geralmente ocorrem no centro cirúrgico destinado para pacientes submetidos ao regime de internação de curta duração (Hospital-Dia). Pelos fatos apresentados, considerou-se como critério de inclusão, nesta etapa, os procedimentos classificados como microneurólise no mapa cirúrgico do Hospital-Dia para de observação presencial.

$\mathrm{Na}$ terceira etapa do projeto, a fim de se determinar o perfil de utilização de medicamentos durante as cirurgias de descompressão de túnel do carpo, no decorrer deum período de dois meses, de julho a setembro de 2019, os procedimentos foram acompanhados virtualmente. Para tal, foram analisadas as fichas de solicitação de medicamentos, referentes a microneurólises. Estas fichas são entregues pelas farmácias satélites dos centros cirúrgicos juntamente com os kits básicos medicamentosos, e os medicamentos não utilizados são devolvidos para o mesmo setor ao final da realização do procedimento cirúrgico, com a ficha atualizada, indicando os itens e as quantidades utilizadas. As movimentações de medicamentos no sistema também foram foco de análise durante esse processo, através de relatórios emitidos no sistema de gestão de informações da instituição.

Uma planilha eletrônica para coleta de dados foi elaborada, onde foram tabulados os dados sobre o uso de medicamentos em cada cirurgia de microneurólise analisada.

O único critério de exclusão atribuído a esta etapa relacionava-se com divergências entre a classificação do procedimento em mapa cirúrgico e em sistema de registro de atendimento. Caso o procedimento constasse como microneurólise no mapa e fosse tipificado de outra forma no sistema de registro, seus dados não eram considerados.

Na quarta etapa do estudo, em outubro de 2019, já com o perfil de uso dos medicamentos analisado, buscou-se estabelecer um elenco específico de medicamentos para a descompressão do túnel do carpo, incluindo aqueles necessários para o bloqueio anestésico e aqueles utilizados como adjuvantes durante a cirurgia, baseando-se em evidências clínicas e científicas. Através destes dados, gerou-se uma nova ficha de solicitação de medicamentos, confeccionada com base no modelo de ficha preexistente, que é dividida em duas seções: a de medicamentos padronizados no kit (previamente preenchida com as quantidades constantes no kit) e a de medicamentos solicitados caso haja necessidade. Esta ficha é fornecida juntamente com o kit básico medicamentoso, e deve ser devolvida à farmácia ao final do procedimento cirúrgico, preenchida de acordo com a utilização de medicamentos.

A quinta etapa do projeto consistiu na realização de comparativo monetário. O kit atual e o proposto foram comparados, considerando seu custo unitário. Para a estimativa de preço, consultou-se o sistema informatizado adotado na instituição, onde se consultaram os dados dos valores dos itens, de acordo com os valores referentes à compra vigente. Além do valor individual de cada kit, estimou-se o custo do uso integral dos procedimentos analisados, visando obter o valor agregado de todas as cirurgias avaliadas, levando em consideração os dois tipos de conjuntos de medicamentos, o atual e o proposto.

Os dados quantitativos foram analisados através de estatística descritiva.

O estudo foi autorizado pela Comissão Científica da instituição, com isenção de submissão a comitê de ética em pesquisa com seres humanos porque trabalhou apenas com dados administrativos, sem acesso a dados pessoais de pacientes ou 
profissionais.

\section{Resultados e Discussão}

Na primeira etapa, foram acompanhados 18 procedimentos de microneurólises presencialmente, no período de 22 a 25 de julho de 2019. Através deste acompanhamento, foi possível melhor compreensão dos aspectos da prática clínica quanto ao uso dos medicamentos, possibilitando o entendimento de particularidades que não se apresentavam suficientemente esclarecidas na literatura.

Os medicamentos utilizados durante o período de observação presencial foram verificados e registrados para fins qualitativos.

$\mathrm{Na}$ segunda etapa, que acompanhou virtualmente os procedimentos, 26 de julho a 20 de setembro de 2019, foram atendidas 102 fichas para cirurgia de microneurólise, porém apenas 76 fichas foram devolvidas à farmácia. A tabela 1 apresenta os dados referentes aos medicamentos identificados em análise prévia presencial e que foram utilizados durante o período de análise virtual, de acordo com as fichas dispensadas e devolvidas nesse período.

Tabela 1: Dados sobre o uso dos medicamentos nas cirurgias observadas presencialmente.

\begin{tabular}{c|c|c|c|c|c|c|c}
\hline ATC & Medicamento & $\begin{array}{c}\text { N. } \\
\text { procedimentos }\end{array}$ & $\begin{array}{c}\text { Média } \pm \text { dp } \\
\text { unidades } \\
\text { utilizadas }\end{array}$ & $\begin{array}{c}\text { Prev. } \\
\text { Med. }\end{array}$ & $\begin{array}{c}\text { Coef. } \\
\text { variação }\end{array}$ & $\begin{array}{c}\% \\
\mathrm{~N}=102\end{array}$ & $\begin{array}{c}\% \\
\mathrm{~N}=76\end{array}$ \\
\hline S02DA01 & $\begin{array}{c}\text { lidocaína } 2 \% \mathrm{sem} \\
\text { vasoconstritor }-5 \mathrm{~mL}\end{array}$ & 72 & $2,3 \pm(1,2)$ & 5 & $55 \%$ & 70,59 & 94,74 \\
\hline N02BB02 & $\begin{array}{c}\text { dipirona } 500 \mathrm{mg} / \mathrm{mL}- \\
2 \mathrm{~mL}\end{array}$ & 63 & $2,6 \pm(1,2)$ & 5 & $46 \%$ & 61,75 & 82,89 \\
\hline N05CD08 & $\begin{array}{c}\text { midazolam } 5 \mathrm{mg} / \mathrm{mL}-3 \\
\mathrm{~mL}\end{array}$ & 59 & $1,0 \pm(0,0)$ & 1 & $0 \%$ & 57,84 & 77,63 \\
\hline A4AA01 & $\begin{array}{c}\text { ondansetrona } 2 \mathrm{mf} / \mathrm{mL}- \\
2 \mathrm{~mL}\end{array}$ & 56 & $1,4 \pm(0,5)$ & 2 & $35 \%$ & 54,9 & 70,68 \\
\hline N01BB09 & $\begin{array}{c}\text { ropivacaína } 1 \%- \\
20 \mathrm{~mL}\end{array}$ & 47 & $1,1 \pm(0,2)$ & 2 & $23 \%$ & 46,08 & 61,84 \\
\hline N01AH01 & $\begin{array}{c}\text { fentanil 0,5 mg/mL - } \\
\mathrm{mL}\end{array}$ & 43 & $1,3 \pm(0,5)$ & 2 & $36 \%$ & 42,16 & 56,58 \\
\hline N01AX10 & $\begin{array}{c}\text { propofol } 10 \mathrm{mg} / \mathrm{mL}-20 \\
\mathrm{~mL}(1 \%)\end{array}$ & 42 & $1,1 \pm(0,3)$ & 2 & $28 \%$ & 41,18 & 55,26 \\
\hline A01AC02 & $\begin{array}{c}\text { dexametasona } 4 \mathrm{mg} / \mathrm{mL}- \\
5 \mathrm{~mL}\end{array}$ & 34 & $1,0 \pm(0,0)$ & 1 & $0 \%$ & 33,33 & 44,74 \\
\hline V03AB25 & flumazenil 0,1 mg/mL & 23 & $1,1 \pm(0,2)$ & 2 & $23 \%$ & 22,55 & 30,26 \\
\hline
\end{tabular}

Legenda: ATC - Anatomical Therapeutic Chemical - classificação segundo o ATC/DDD Index (www.whocc.no/atcddd); Média \pm dp unidades utilizadas - média \pm desvio padrão de unidades utilizadas; Prev. Med. - previsão medicamentosa; Coef. Variação coeficiente de Variação; \% N = 102 - valor em porcentagem considerando o valor da amostra 102; \% N = 76 - valor em porcentagem considerando o valor da amostra 76.

Fonte: Autores (2019).

Neste período, a apuração era realizada antes do horário de funcionamento dos centros cirúrgicos, verificando-se a programação de cirurgias alvo, na data em questão, em todas as salas de centro cirúrgico da instituição. Assim, todas as microneurólises realizadas na instituição foram incluídas na análise, sendo foi possível obter mais dados no período do estudo, minimizando subestimativas possíveis.

Segundo as movimentações de estoque dos medicamentos utilizados, registradas no sistema informatizado, 17 procedimentos foram realizados no centro cirúrgico de pacientes de regime de longa duração, enquanto 85 foram realizados no 
centro cirúrgico de regime de curta duração. Entretanto, observou-se que algumas cirurgias foram realocadas e seus registros se mantiveram em locais de origem, indicando que pode haver divergência em relação ao local de realização da cirurgia.

Em 26 procedimentos, as fichas de solicitação de medicamentos não foram devolvidas às farmácias satélites dos centros cirúrgicos, correspondendo a 25,49\% das 102 cirurgias analisadas. Desta forma, houve subestimativa quanto à real utilização de medicamentos correspondente a cerca de um quarto das cirurgias avaliadas. Com isso, também se constatou a inviabilidade do rastreamento dos medicamentos.

Para 2 procedimentos existia mais de uma movimentação de medicamentos do estoque para a mesma cirurgia, ou seja, para cada um destes, havia dois registros de movimentação de medicamentos do estoque. Constatando-se que, para cada caso, houve devolução de duas fichas de solicitação para o mesmo procedimento cirúrgico, em momentos diferentes do mesmo dia.

\subsection{Inclusão ou exclusão de medicamentos padrão}

O kit atual para todas as cirurgias elenca 24 itens, dos quais 23 tem seu consumo registrado em sistema. O vigésimo quarto item desta lista, água destilada, não é movimentada em estoque. Dentre a lista de medicamentos padrão e que tem seu consumo lançado em sistema, somente 12 constam como utilizados em maior frequência (15\% ou mais vezes). Quatro itens desta lista não foram utilizados em nenhum dos procedimentos cirúrgicos analisados, sendo eles: bupivacaína $0,5 \%$ + glicose; levobupivacaína isobárica $0,5 \%$; metoclopramida; e succinilcolina.

Os dados sobre a utilização dos medicamentos que compõem o kit atual indicaram que a oferta medicamentosa excede a necessidade durante a realização de microneurólises. Com a revisão destes itens, buscou-se obter a modificação do cenário então instalado, a fim de adequá-lo ao perfil real de utilização da cirurgia.

A bupivacaína $0,5 \%$ + glicose consta entre os medicamentos não utilizados em nenhum dos 76 procedimentos em que se observou movimentação de consumo de estoque. Sua indicação, descrita em bula, é para realização de bloqueio subaraquinoideo (Neocaína Pesada ${ }^{\circledR}, 2018$ ). No cenário de estudo, a técnica cirúrgica para descompressão do túnel do carpo utiliza a anestesia local, procedimento menos complexo. Assim, observou-se a incompatibilidade de indicações que, somada à não utilização da bupivacaína $0,5 \%$ + glicose durante o período de estudo, gera indícios para que seja considerada a remoção de tal item na formulação do kit proposto.

A levobupivacaína isobárica $0,5 \%$, apesar de indicada para bloqueio local, também figura entre os itens não utilizados (Novabupi ${ }^{\circledR}$, 2018). A subutilização deste medicamento pode estar diretamente relacionada ao desabastecimento dele na instituição. No entanto, a ausência deste medicamento não acarretou prejuízos durante a realização do procedimento em estudo, indicando que ele é facilmente substituível. Seu desabastecimento, aliado à sua fácil substituição, indicam que este medicamento pode ser retirado do kit sem maiores prejuízos.

A levobupivacaína $0,5 \%$ e a bupivacaína isobárica $0,5 \%$ são anestésicos locais que constituíram o rol dos medicamentos usados. O primeiro medicamento foi utilizado em 5,26\% das cirurgias, enquanto o segundo foi empregado em $3,95 \%$ dos casos.

Os anestésicos locais mais utilizados foram a lidocaína $2 \%(94,74 \%)$ e a ropivacaína $1 \%(61,84 \%)$. Quando comparados com o uso dos anestésicos mencionados anteriormente, juntamente com o conhecimento clínico adquirido, verifica-se que os anestésicos de escolha para microneurólises coincidem com os mais empregados durante a pesquisa. Estes medicamentos, habitualmente, são utilizados em associação para a realização do bloqueio anestésico local.

Caso seja demonstrada necessidade de padronização de mais medicamentos anestésicos para a realização da descompressão do túnel do carpo, considerações devem ser feitas acerca da bupivacaína e da levobupivacaína. No que tange o custo do item, segundo pesquisa de preço no sistema da própria instituição, o primeiro medicamento apresenta, como vantagem, o menor valor. No entanto, ao ser considerada a segurança dos medicamentos, o segundo se mostra superior, devido 
à exclusividade do isômero levogiro deste fármaco. Este apontamento é justificado pela cardiotoxicidade atribuída aos isômeros dextrogiros das aminas anestésicas (Nociti, 2017).

A metoclopramida também está entre os medicamentos padronizados e não utilizados no kit básico original. Quando comparado ao outro medicamento disponível no kit, com indicação para o tratamento de náusea e vômito, a metoclopramida não parece ser o medicamento de escolha durante a cirurgia. Os números expressados durante o período de observação apontam para a ondansetrona como medicamento mais empregado nesses casos. Desta forma, caso se comprove necessidade, os números obtidos durante o estudo devem ser levados em conta para a padronização de um único medicamento (Abreu et al., 2006), com prioridade para o segundo, que foi aquele com uso constatado durante o estudo.

Apesar da ampla utilização de medicamentos destinados ao tratamento de náusea e vômito no pré-operatório, não há evidência científica significativa sobre a ocorrência de destes agravos pós-operatório (NVPO) na utilização de anestesia local. Tal comorbidade parece estar mais diretamente relacionada à anestesia geral. Como estratégia para a redução dos riscos deste quadro é preconizado hidratar adequadamente o paciente, além de dar preferência da aplicação da anestesia local/bloqueio periférico (Braga, 2017).

A succinilcolina também é um item que compõe o kit medicamentoso padrão atual e que não foi utilizado em nenhum procedimento observado durante o período do estudo. Segundo a bula, este é um relaxante muscular esquelético, indicado como adjuvante da anestesia geral, para facilitar a intubação traqueal e proporcionar relaxamento do músculo esquelético durante a cirurgia ou ventilação mecânica (Succinil Colin®, 2018). Uma vez que tenha sido verificada divergência entre sua indicação e as práticas ocorridas durante o procedimento observado, este medicamento não deve ser integrante do novo kit de medicamentos.

De acordo com as informações adquiridas através da planilha de coleta de dados, juntamente com dados da literatura e o observado na prática clínica, verificou-se que a sedação na microneurólise é realizada pela associação de um benzodiazepínico com um opióide.

O opióide de escolha para realização de sedação durante os procedimentos foi o fentanil, utilizado em $75 \%$ dos casos. Apesar de sua apresentação com $5 \mathrm{~mL}$ ser a padronizada para o kit em questão, deve ser avaliada a possibilidade de troca de padronização, retirando o item padronizado atualmente e inserindo a apresentação de $2 \mathrm{~mL}$. Tal sugestão se deve a maior utilização da apresentação de menor volume (56,58\%) quando comparada com a de maior volume (23,68\%).

Analisando o uso de benzodiazepínicos, observou-se que o representante da classe mais utilizado desta classe foi o midazolam, sendo usado em $77,63 \%$ das cirurgias. A semelhança entre as porcentagens de utilização de opioides e benzodiazepínicos confirmam que a sedação é realizada através da associação destas duas classes farmacológicas, através destes dois representantes.

Mais de um medicamento destinado ao tratamento ou prevenção da hipotensão arterial associada à anestesia intratecal, epidural e anestesia geral está padronizado no kit atual, sendo eles: efedrina, epinefrina e etilefrina. Estes medicamentos também são indicados ao tratamento do choque, situação clínica de queda abrupta e grave da pressão arterial e que não responde com o tratamento com reposição de fluidos administrados na veia (Efedrinß, 2018; Adren®, 2019; Etilefrilß, 2018).

Apesar das comorbidades acima não serem complicações comuns aos casos em estudo, três são os medicamentos padronizados no kit. Caso seja verificada a necessidade da permanência de um fármaco com esta finalidade, os dados apresentados na tabela de coleta de dados devem ser levados em consideração.

Considerando os itens destinados ao tratamento da hipotensão que compõem o kit atual, somente a efedrina foi utilizada de forma significativa, sendo usada em $17,11 \%$ dos procedimentos. Portanto, deve ser considerada a manutenção somente deste item no novo conjunto de medicamentos. Esta providência auxiliaria ainda na diminuição de confusões relativas 
a medicamentos de nomes parecidos.

A atropina é outro medicamento padronizado no kit atual. Utilizada em $28,95 \%$ dos procedimentos analisados, é indicada nos casos de intercorrências em função de arritmias ou bradicardia sinusal severa e síncope devido à hiperatividade do reflexo sino-carotídeo, no controle do bloqueio cardíaco atrioventricular decorrente de um aumento da atividade vagal (Atropion®, 2019).

A considerável frequência de uso da atropina aponta para duas possibilidades de desfecho igualmente preocupantes: seu uso indevido; ou o excesso de intercorrências tratáveis por este medicamento nas cirurgias analisadas. Apesar de respostas inconclusivas sobre a utilização dele, o caráter de medicamento de emergência indica a manutenção deste fármaco no novo kit.

Os medicamentos com finalidade profilática da aspiração de conteúdo gástrico também parecem ser utilizados de maneira inadequada. Esta incoerência está relacionada ao tipo de anestesia utilizada neste procedimento, sendo estes indicados como profilaxia em cirurgias em que os pacientes são submetidos à anestesia geral e em pacientes de risco (Cloridrato de Ranitidina EMS®, 2019; Omeprazol Sódico CRISTÁLIA®, 2019). Apesar disto, nas microneurólises analisadas, estes também foram amplamente utilizados.

O omeprazol, medicamento do kit atual, foi utilizado em $28,95 \%$. Já quando se observa a ranitidina, item que não compõe o elenco de medicamentos do conjunto dispensado atualmente, seu uso foi de $14,47 \%$. O percentual agregado de utilização foi de $38,16 \%$ dos casos, valor bastante alto para medicamentos sem indicação.

Em quatro procedimentos o omeprazol e a ranitidina parecem ter sido administrados ao paciente, conduta que não parece justificada. Caso seja comprovada a necessidade da manutenção de um medicamento para este fim, levando em consideração a composição do kit atual, apenas um destes medicamentos deveria ser mantido no novo kit. Nesse sentido, seria mais apropriado manter o omeprazol, que já faz parte do kit atual, e que também figura como o mais utilizado.

A dexametasona é o medicamento da classe dos glicocorticoides padronizado no kit atual, e foi utilizado em 44,74\% dos procedimentos, se mostrando como um fármaco amplamente utilizado nas cirurgias de descompressão.

Algumas dúvidas surgiram acerca da necessidade de utilização deste medicamento. Sua classe medicamentosa normalmente é utilizada em infiltrações locais, visando aliviar as dores causadas pela compressão do nervo. Nos casos em que a compressão do nervo se mostra tão agravada, se fazendo necessária intervenção cirúrgica, não foram observadas evidências na literatura sobre indicações de realização de infiltração (Pereira et al., 2005).

Caso seja comprovada a necessidade da manutenção de medicamentos para a infiltração, as evidências científicas apontam para melhores resultados com a utilização de hidrocortisona (Hollander, 1970), medicamento também padronizado na instituição. Assim, dever-se-ia analisar a viabilidade da alteração entre estes dois medicamentos no kit novo.

Outra finalidade da dexametasona é a prevenção de NVPO. Para este quadro clínico, como já mencionado anteriormente, a melhor eficácia, segundo literatura, é demonstrada através do uso da ondansetrona (Abreu et al., 2006).

A única aplicação da dexametasona que justifica sua manutenção no novo conjunto de medicamentos padronizados é a sua aplicação no pré-operatório, com o objetivo de modular o edema pós-operatório (Vicente et al., 2013).

O parecoxibe e a dipirona são medicamentos com indicação analgésica, utilizados para este fim neste tipo de procedimento, padronizados no elenco do kit atual. O primeiro foi utilizado em $43,42 \%$ dos procedimentos. Já o segundo teve uma frequência muito maior, sendo usado em $82,89 \%$.

Deve ser avaliada a necessidade de manutenção de ambos os medicamentos e sua a utilização. A dipirona, medicamento mais usado, parece atender consideravelmente às necessidades. Este, segundo dados obtidos no sistema de gestão, também é o medicamento mais barato, tendo custo 137 vezes menor que o parecoxibe. Tais informações são significativamente relevantes para consideração da manutenção somente deste no kit padrão, mantendo o outro como possibilidade de solicitação, caso haja necessidade. 
A neostigmina é um fármaco que merece ser cuidadosamente analisado, dentre os medicamentos utilizados. Sua baixa utilização deve ser fator importante ao se considerar a retirada do mesmo entre os componentes do kit básico medicamentoso destinado às microneurólises. Aplicado em casos de constipação atônica, meteorismo (por exemplo, antes do exame radiológico); atonia intestinal pós-operatória e retenção urinária; miastenia gravis pseudoparalítica; antagonista dos curarizantes (para neutralizar o efeito miorrelaxante do curare e dos preparados do mesmo tipo) (Metilsulfato de Neostigmina Blau $\left.{ }^{\circledR}, 2018\right)$, este medicamento foi utilizado somente em um procedimento. Uma vez que não são utilizados medicamentos a base de curare durante o procedimento e que as complicações citadas não são comuns em microneurólises, recomenda-se a retirada deste item do kit.

A lidocaína geleia é um medicamento padronizado no kit vigente e que aparece como utilização insignificante. Em todo caso, deve ser considerada a manutenção deste medicamento na proposta do novo kit. A justificativa para tal é a sua apresentação multidose, possibilitando a utilização do mesmo item por diversas vezes. Este fato pode acarretar uma subestimação da frequência de uso deste medicamento. Por isto, considera-se necessário manter o medicamento.

Outro item que tem sua utilização subestimada é a água bidestilada. Isto ocorre porque este medicamento não tem registro de consumo no sistema. Por isto, apesar de ampla utilização, esta não aparece entre os dados levantados no sistema de gestão institucional. Assim, opta-se pela manutenção do item na formulação do novo conjunto de medicamentos.

\subsection{Inclusão ou exclusão de medicamentos não padrão}

Ao todo, foram solicitados durante os procedimentos observados 23 diferentes itens não constantes como padrão no kit atual (Tabela 2). Dentre estes itens, somente o flumazenil, o fentanil e o propofol foram utilizados com frequência significativa, quando considerados os padrões de corte do estudo. 
Tabela 2: Dados dos itens não padronizados no kit atual, utilizados durante os procedimentos observados.

\begin{tabular}{|c|c|c|c|c|c|c|c|}
\hline ATC & Medicamento & $\begin{array}{c}\mathrm{N}^{\circ} \\
\text { proced. }\end{array}$ & $\begin{array}{c}\text { Média } \pm \mathrm{dp} \\
\text { unidades } \\
\text { utilizadas }\end{array}$ & Prev. Med. & $\begin{array}{l}\text { Coef. } \\
\text { variação }\end{array}$ & $\% \mathrm{~N}=102$ & $\% \mathrm{~N}=76$ \\
\hline N01AH01 & fentanil $0,5 \mathrm{mg} / \mathrm{mL}-2 \mathrm{~mL}$ & 43 & $1,3 \pm(0,5)$ & 2 & $36 \%$ & 42,16 & 56,58 \\
\hline N01AX10 & propofol $10 \mathrm{mg} / \mathrm{mL}-20 \mathrm{~mL}(1 \%)$ & 42 & $1,1 \pm(0,3)$ & 2 & $28 \%$ & 41,18 & 55,26 \\
\hline V03AB25 & flumazenil $0,1 \mathrm{mg} / \mathrm{mL}-5 \mathrm{~mL}$ & 23 & $1,1 \pm(0,2)$ & 2 & $23 \%$ & 22,55 & 30,26 \\
\hline A02BA02 & ranitidina $25 \mathrm{mg} / \mathrm{mL}-2 \mathrm{~mL}$ & 11 & $1,0 \pm(0,0)$ & 1 & $0 \%$ & 10,78 & 14,47 \\
\hline J01DC02 & cefuroxima $750 \mathrm{mg} \mathrm{IV}$ & 9 & $1,8 \pm(0,4)$ & 3 & $25 \%$ & 8,82 & 11,84 \\
\hline N01AX03 & $\begin{array}{c}\text { dextrocetamina cloridrato } 50 \\
\mathrm{mg} / \mathrm{mL}-2 \mathrm{~mL}\end{array}$ & 9 & $1,0 \pm(0,0)$ & 1 & $0 \%$ & 8,82 & 11,84 \\
\hline $\mathrm{C} 02 \mathrm{AC} 01$ & clonidina $150 \mathrm{mg} / \mathrm{mL}$ - $1 \mathrm{~mL}$ & 5 & $1,0 \pm(0,0)$ & 1 & $0 \%$ & 1,96 & 2,63 \\
\hline N01AX10 & propofol $50 \mathrm{~mL}(1 \%)$ & 3 & $1,0 \pm(0,0)$ & 0 & $0 \%$ & 2,94 & 3,95 \\
\hline N01AB08 & sevoflurano $100 \% 250 \mathrm{~mL}$ & 3 & $1,0 \pm(0,0)$ & 1 & $0 \%$ & 2,94 & 3,95 \\
\hline M01AE03 & cetoprofeno IV $100 \mathrm{mg}$ & 2 & $1,0 \pm(0,0)$ & 0 & $0 \%$ & 1,96 & 2,63 \\
\hline R01AX10 & cloreto de sódio $0,9 \%-10 \mathrm{~mL}$ & 2 & $1,5 \pm(0,7)$ & 3 & $47 \%$ & 1,96 & 2,63 \\
\hline N05BA01 & diazepam $5 \mathrm{mg} / \mathrm{mL}-2 \mathrm{~mL}$ & 2 & $1,0 \pm(0,0)$ & 1 & $0 \%$ & 1,96 & 2,63 \\
\hline H02АB09 & hidrocortisona $500 \mathrm{mg}$ & 2 & $1,0 \pm(0,0)$ & 1 & $0 \%$ & 1,96 & 2,63 \\
\hline M03AC09 & rocurônio $10 \mathrm{mg} / \mathrm{mL}-5 \mathrm{~mL}$ & 2 & $1,0 \pm(0,0)$ & 1 & $0 \%$ & 1,96 & 2,63 \\
\hline B02AA02 & ácido tranexâmico - $5 \mathrm{~mL}$ & 1 & $4,0 \pm(0,0)$ & 0 & $0 \%$ & 0,98 & 1,32 \\
\hline B05XA02 & $\begin{array}{l}\text { bicarbonato de sodio } 8,4 \% \\
\text { sol injetavel - } 10 \mathrm{~mL}\end{array}$ & 1 & $1,0 \pm(0,0)$ & 0 & $0 \%$ & 0,98 & 1,32 \\
\hline C09AA01 & captopril $25 \mathrm{mg}$ comprimido & 1 & $1,0 \pm(0,0)$ & 0 & $0 \%$ & 0,98 & 1,32 \\
\hline N05CM18 & dexmedetomidina $100 \mathrm{mcg} / \mathrm{mL}$ & 1 & $1,0 \pm(0,0)$ & 0 & $0 \%$ & 0,98 & 1,32 \\
\hline H02AB04 & metilprednisolona $80 \mathrm{mg}$ f/a & 1 & $1,0 \pm(0,0)$ & 0 & $0 \%$ & 0,98 & 1,32 \\
\hline N02AF02 & nalbufina $10 \mathrm{mg} / \mathrm{mL}-1 \mathrm{~mL}$ & 1 & $1,0 \pm(0,0)$ & 0 & $0 \%$ & 0,98 & 1,32 \\
\hline V03AB35 & sugamadex $100 \mathrm{mg} / \mathrm{mL}-2 \mathrm{~mL}$ & 1 & $1,0 \pm(0,0)$ & 0 & $0 \%$ & 0,98 & 1,32 \\
\hline N02AX02 & tramadol $100 \mathrm{mg} / 2 \mathrm{~mL}$ & 1 & $1,0 \pm(0,0)$ & 0 & $0 \%$ & 0,98 & 1,32 \\
\hline N02AX02 & tramadol $50 \mathrm{mg} / \mathrm{mL}-1 \mathrm{~mL}$ & 1 & $1,0 \pm(0,0)$ & 0 & $0 \%$ & 0,98 & 1,32 \\
\hline
\end{tabular}

Legenda: ATC - Anatomical Therapeutic Chemical - classificação segundo o ATC/DDD Index (www.whocc.no/atcddd); Média \pm dp unidades utilizadas - média \pm desvio padrão de unidades utilizadas; $\mathrm{N}^{\circ}$ proced. - número de procedimentos; Prev. Med. - previsão medicamentosa; Coef. Variação - coeficiente de Variação; \% N = 102 - valor em porcentagem considerando o valor da amostra 102; $\% \mathrm{~N}=76$ - valor em porcentagem considerando o valor da amostra 76.

Fonte: Autores (2019).

O flumazenil tem, dentre suas indicações, a promoção da reversão completa ou parcial dos efeitos sedativos centrais dos benzodiazepínicos, podendo ser utilizado em cirurgias procedimentos que envolvam anestesia geral, procedimentos diagnósticos e terapêuticos de curta duração em pacientes hospitalizados e de ambulatório (Flumazenil União Químicaß, 2019). Esta indicação explicita a necessidade da introdução deste item no conjunto de itens padronizados.

O propofol é indicado para a manutenção da sedação (Provive®, 2018). Apesar de não constar no kit, a prática clínica demonstrou que este medicamento é dispensado para todas as cirurgias realizadas, devendo assim ser considerado como item padronizado na atualização do kit.

O fentanil, em sua apresentação de $2 \mathrm{~mL}$, é o último dos três itens não padronizados que foram amplamente utilizados. Acerca deste fármaco, já se discutiu sobre sua utilização e padronização anteriormente. 


\subsection{Utilização incomum de medicamentos}

Durante análise dos dados, observou-se que alguns medicamentos utilizados não eram compatíveis com o perfil cirúrgico ou com o próprio perfil de utilização deles. Assim, verificou-se a necessidade de comentar os casos neste trabalho.

Dentre as atipicidades apresentadas no uso de medicamento está a utilização de um comprimido de captopril $25 \mathrm{mg}$ durante o procedimento cirúrgico. Esta não é a forma farmacêutica adequada para o manejo de quadro hipertensivos intraoperatórios. Tais casos devem ser manejados pela administração de líquidos intravenosos (Do Carmo \& Caramelli, 2012).

A administração deste medicamento se mostrava injustificada mesmo em seu uso por via sublingual, para a redução da pressão arterial, uma vez que consistia em um comprimido de captopril para administração entérica. No elenco de itens passiveis de solicitação, encontram-se outros medicamentos que poderiam ser utilizados de forma mais adequada, visando à segurança do paciente e a consecução do objetivo a ser atingido.

Em três casos, verificou-se registro de uso de sevoflurano. Sendo este um medicamento inalatório, destinado à indução e manutenção de anestesia, acredita-se na sua utilização ou registro em sistema equivocado. A hipótese mais provável é que tenha se tratado de um caso de registro equivocado no sistema, uma vez que o protocolo anestésico da referida cirurgia envolve anestesia local, não sendo necessária, desta forma, a utilização de gases inalatórios.

Em um dos 76 casos cirúrgicos aos quais foram atribuídos medicamentos, houve a utilização do sugamadex, que é indicado para proporcionar a reversão do bloqueio neuromuscular induzido por rocurônio ou vecurônio (Bridion $®$, 2018). No entanto, não houve a utilização de nenhum medicamento derivado de curare durante o mesmo procedimento, dificultando a possível explicação da utilização do primeiro medicamento citado. Durante o procedimento em questão, foi utilizada a dextrocetamina. Este foi o único medicamento utilizado como adjuvante anestésico e que não está direcionado para a anestesia local. Apesar disto, não é justificada a utilização do sugamadex, uma vez que a dextrocetamina não causa o relaxamento muscular causado pelos cumarínicos e alvo da reversão deste medicamento. Fato que indica também a possibilidade de registro equivocado no sistema de gestão.

Nas duas cirurgias nas quais houve a utilização de derivados do curare, sendo o rocurônio o fármaco desta classe padronizado no kit atual, o sugamadex não foi utilizado. A utilização desta classe medicamentosa para este tipo de cirurgia causa estranheza, já que são indicados como adjuvantes à anestesia geral para facilitar a intubação traqueal em procedimentos de rotina e de indução de sequência rápida de anestesia, bem como para relaxar a musculatura esquelética durante intervenções cirúrgicas (Bricola et al., 2018).

Devido à sua aplicabilidade, a utilização de rocurônio em microneurólises é motivo de alerta. Durante procedimentos realizados com sucesso, não deveria se fazer necessário o relaxamento muscular do paciente, mostrando assim que estes procedimentos podem ter sido marcados por intercorrências. Os casos em que não são observadas complicações são ainda mais chamativos, uma vez que esta é a indicação para qual este medicamento foi utilizado de forma não justificada.

Em uma das cirurgias, houve a associação de dois opioides, sendo eles fentanil e nalbufina. O primeiro consta como medicamento protocolar deste tipo de cirurgia. O segundo precisa ser solicitado para ser administrado, uma vez que não consta como medicamento padrão do kit atual.

Segundo consulta ao Fentanyl Interactions Checker (n.d.), a associação destes dois opioides é contraindicada, uma vez que podem resultar em sedação profunda, depressão respiratória, coma e morte. Além disto, a nalbufina também pode anular o efeito analgésico do primeiro fármaco citado (Benseñor \& Cicarelli, 2003).

A dextrocetamina é indicada como anestésico único em intervenções diagnósticas e cirúrgicas que não necessitem de relaxamento muscular (Ketamin® , 2018). Sua indicação como anestésico geral diminui significativamente o número de vezes em que precisa ser utilizada no procedimento cirúrgico em questão.

Apesar do disposto anteriormente, este medicamento foi utilizado em nove $(11,84 \%)$ de todos os procedimentos 
cirúrgicos realizados. Estes mesmos procedimentos também utilizaram lidocaína, indicando que estes podem ter sido iniciados com anestesia local. Tal afirmação é corroborada pela utilização de ropivacaína ou bupivacaína em oito dos nove procedimentos. O midazolam, benzodiazepínico utilizado na sedação deste tipo de procedimento também foi utilizado nesses nove procedimentos. O fentanil, que normalmente é utilizado em associação com o midazolam, foi utilizado em sete das nove cirurgias. Já o propofol, comumente utilizado para a manutenção da sedação, foi utilizado seis vezes.

É possível que intercorrências tenham acontecido durante a realização dos procedimentos, necessitando a utilização de anestesia geral, o que justificaria a utilização da dextrocetamina. Os dados podem corroborar com esta afirmação, uma vez que em um dos procedimentos, foram associadas dextrocetamina e dexmedetomidina, além da utilização dos medicamentos usualmente empregados no bloqueio local anestésico.

O ácido tranexâmico é destinado para o controle e a prevenção de sangramentos provocados por cirurgias, traumatismos e doenças com tendência a sangramentos (Ácido Tranexâmico Blauß, 2018). Devido à sua aplicabilidade, a presença deste medicamento dentre os itens empregados em um dos procedimentos realizados causa estranheza, justificada pela utilização do torniquete durante o procedimento cirúrgico, técnica utilizada para este mesmo fim cirúrgico.

O bicarbonato de sódio, utilizado somente uma vez, é indicado na parada cardíaca, acidose metabólica nos casos de diabetes, diarreia, intoxicações por ácidos exógenos ao organismo (bórico e salicílico), retenção de ácidos não voláteis, uremia, acidose lática (Bicarbonato de sódio Hypofarma®, 2018). Através da análise dos outros medicamentos utilizados durante este procedimento, acredita-se que houve intercorrências nesta cirurgia, justificando a sua administração.

Em seis procedimentos cirúrgicos, o medicamento flumazenil foi utilizado sem o uso prévio de benzodiazepínicos, indicando assim uma possível utilização equivocada. Não foi encontrada, em literatura, qualquer justificativa que explicasse satisfatoriamente a empregabilidade de tal fármaco.

Em dois procedimentos, foram utilizados, em associação, midazolam e diazepam. Também neste caso, não foram encontradas evidências que comprovassem efeito benéfico na associação destes medicamentos.

Em uma das cirurgias, usou-se, no mesmo procedimento, etilefrina e clonidina, causando certa confusão acerca do entendimento sobre as circunstâncias da administração destes medicamentos. Isto ocorre porque os dois medicamentos têm indicações opostas. A única justificativa plausível para o caso seria a utilização da clonidina como medicamento adjuvante da analgesia. Apesar deste uso ser viável, não costuma ser corriqueiro durante microneurólises, já que seu uso em associação a anestésicos locais ocorre mediante necessidade de analgesia intratecal ou epidural. No entanto, uma queda brusca da pressão arterial, relacionada à administração da clonidina, poderia justificar a necessidade de uso da etilefrina (Clonidin®, 2019).

A cefazolina, antibiótico da classe das cefalosporinas, foi utilizada em 15 (19,74\%) dos procedimentos analisados. Apesar de sua ampla aplicação como antibioprofilaxia, os respectivos protocolos para pacientes cirúrgicos não apontam indicação do mesmo, ou de qualquer outro antibiótico, em cirurgias ortopédicas eletivas sem implante (Hospital Sírio-Libanês, 2015). Desta forma, foi observada a utilização deste medicamento sem que haja evidência cientifica que comprove a eficácia do mesmo para estas situações.

Em cirurgias eletivas com implante ou manipulação óssea, a indicação da antibioticoprofilaxia é de $2 \mathrm{~g}$ EV de cefazolina na indução da cirurgia, $1 \mathrm{~g} 4 / 4 \mathrm{~h}$ intraoperatório. Já no pós-operatório, indica-se administrar 1g 8/8h durante $24 \mathrm{~h}$. Apesar de não haver manipulação óssea ou mesmo implante durante este tipo de procedimento, caso a equipe optasse pela utilização deste protocolo antibioticoprofilático, considerando a curta duração da cirurgia, a dose utilizada de cefazolina deveria ser de 2 gramas EV, referente ao medicamento aplicado somente durante a indução. Não seria necessária a administração de outras doses, referentes àquelas utilizadas durante o intraoperatório (Hospital Sírio-Libanês, 2015).

No entanto, dois dos procedimentos cirúrgicos analisados apontaram divergências no seguimento do protocolo. Em um deles, o paciente recebeu somente 1 grama de cefazolina, o que configura uma subdose do tratamento, expondo o paciente 
a riscos, como o de resistência aos antibióticos.

$\mathrm{O}$ outro procedimento, onde foi observada divergência do protocolo proposto, registrou o uso de 5 gramas de cefazolina. Este caso somente se justificaria, levando em conta o protocolo cirurgias eletivas com implante ou manipulação óssea, ainda com o uso equivocado, caso houvesse alguma intercorrência que justificasse estender cirurgia por mais doze horas.

A cefuroxima também é uma cefalosporina normalmente utilizada como antibioticoprofilaxia. Consiste na segunda alternativa profilática, como consta no protocolo institucional. Se tratando dos procedimentos avaliados, este antibiótico foi utilizado em nove cirurgias, também se enquadrando na utilização indevida da antibioticoprofilaxia, uma vez que sua indicação é destinada somente para cirurgias envolvendo próteses. Nestas, são indicados 1,5 grama EV deste medicamento no momento da indução, $750 \mathrm{mg}$ 4/4h intraoperatório e $750 \mathrm{mg}$ 8/8h no pós-operatório, por uma duração de 24 horas (Hospital SírioLibanês, 2015).

Assim como no uso da cefazolina, foram observadas divergências na dose protocolar do medicamento. Em duas cirurgias nas quais a cefuroxima foi utilizada, a dose administrada foi de somente $750 \mathrm{mg}$, configurado uma subdose do tratamento, expondo o paciente a riscos, como o de resistência aos antibióticos.

Os dois medicamentos citados anteriormente estavam com matéria prima em falta no mercado à época do estudo. Seja por desabastecimento, seja pelos altos preços aos quais eram ofertados, grande era a dificuldade em adquiri-los. Considerando estes fatos, juntamente com o perfil da instituição, um instituto referência em traumatologia e ortopedia, o uso indevido destes poderia acarretar danos tanto econômicos quanto processuais.

Algumas técnicas, visando menor dor pós-operatória, são utilizadas. Dentre elas, se encontra a analgesia preemptiva, que consiste em um tratamento farmacológico iniciado antes do procedimento cirúrgico, a fim de prevenir a hiperalgesia (DEJEAN et al., 2008).

Em $30(39,47 \%)$ procedimentos observados, administrou-se, em associação, dipirona e parecoxibe. Não foram encontradas evidências que comprovassem que a associação destes dois trouxesse benefícios em relação à administração exclusiva de um deles (Garcia et al., 2001).

Alguns estudos abordam a utilização eficiente da associação entre dexametasona e anti-inflamatórios não esteroidais (AINE) para a analgesia preemptiva. Tal associação foi realizada em 20 (26,32\%) dos procedimentos em estudo, pela associação de dexametasona com parecoxibe. No entanto, a eficácia da analgesia parece ser variante de acordo com o tipo de cirurgia, não havendo evidências que comprovem que este método funcione tão bem para microneurólises quanto para as cirurgias dentárias, as quais demonstraram efetividade (Bauer et al., 2013; Aida et al., 1999).

Ainda no campo da ortodontia, observam-se efeitos positivos na associação da dexametasona com a dipirona. Tal combinação mostrou resultados interessantes acerca da diminuição da hipersensibilidade do nervo alveolar lingual e inferior, após extrações de terceiros molares. Apesar de não terem sido encontradas evidências sobre a eficiência na microneurólise, a dexametasona foi associada à dipirona em trinta das cirurgias analisadas (Barron et al., 2004).

Anteriormente, foram citados casos de sucesso entre a associação da dexametasona com AINE ou com a dipirona. Tais casos não se relacionavam diretamente ao procedimento de estudo, tão pouco à associação das três classes. No entanto, em dezenove procedimentos, foram associados parecoxibe, dexametasona e dipirona (Righeti \& Lauretti, 2014).

Em três procedimentos cirúrgicos, foram utilizadas as duas apresentações de propofol: uma de $20 \mathrm{~mL}$ e outra de 50 $\mathrm{mL}$. Tal fato pode apontar para o desperdício do medicamento, que poderia ocorrer no caso da utilização incompleta da apresentação de $50 \mathrm{~mL}$. Qualquer volume abaixo de $40,1 \mathrm{~mL}$ utilizado de tal frasco, poderia ser convertido pelo uso das apresentações de $20 \mathrm{~mL}$.

A administração de consideráveis volumes de propofol também devem ser avaliadas clinicamente, de acordo com 
cada paciente. Deve-se levar em conta a dose máxima deste medicamento em adultos, $12 \mathrm{mg} / \mathrm{kg} / \mathrm{hora}$, levando em consideração a concentração de $10 \mathrm{mg} / \mathrm{mL}$ do produto padronizado na instituição (Provive ${ }^{\circledR}, 2018$ ).

\subsection{Novo kit cirúrgico proposto}

Como quarta etapa do projeto, o estabelecimento de um kit medicamentoso é realizado após estudos referentes à prática em questão, considerando ainda o perfil de utilização de medicamentos verificado na etapa anterior.

Quando se tem por objetivo criar um kit medicamentoso para um tipo de cirurgia, por exemplo, é necessária a avaliação de todo o protocolo cirúrgico, quantificando e qualificando os medicamentos utilizados. Também é necessário levar em consideração a opinião do corpo clínico, possibilitando colher informações sobre o procedimento que não se faz claro a quem não esteja diretamente envolvido na prática cirúrgica.

Uma vez coletadas todas as informações pertinentes, se faz viável a comparação entre o que já existe e o que se deseja implantar, visando o estabelecimento da proposta para um novo kit básico medicamentoso específico para procedimentos de microneurólise. Assim, com os ajustes necessários, propõe-se um novo elenco padrão, que tem como meta a redução de custos e a mitigação dos erros de medicação, contribuindo também para o aumento da segurança do paciente.

Assim, a partir da análise criteriosa da utilização de medicamentos nas cirurgias de descompressão do nervo mediano observadas, identificamos o perfil de utilização de medicamentos. Com as frequências e quantidades verificadas, gerou-se média e desvio padrão para cada medicamento utilizado, medidas de dispersão que foram essenciais para a decisão qualitativa e quantitativa de medicamentos a constarem no novo kit proposto.

Alguns medicamentos foram relacionados como necessários para o conjunto de medicamentos, apesar de apresentarem perfil diferente daqueles medicamentos essenciais para a realização deste tipo de procedimento cirúrgico. Isto se deve a precauções tomadas, considerando alguns eventos adversos, intercorrências e emergências possíveis durante o procedimento.

Também foram introduzidos na proposta do novo kit medicamentos amplamente utilizados, para os quais foram verificadas evidências científicas que comprovassem sua indicação. O Quadro 1 apresenta a comparação entre os elencos do kit atual e do kit proposto. 
Quadro 1: Comparação dos elencos entre o kit atual e o novo kit proposto.

\begin{tabular}{|c|c|c|c|}
\hline \multirow{2}{*}{ ATC } & \multirow{2}{*}{ Medicamento } & \multicolumn{2}{|c|}{ Quantidade (unidade) } \\
\hline & & Kit Atual & Kit Proposto \\
\hline J02AA01 & água bidestilada estéril - $10 \mathrm{~mL}$ & 9 & 9 \\
\hline A03BA01 & atropina $0,25 \mathrm{mg} / \mathrm{mL}-1 \mathrm{~mL}$ & 10 & 10 \\
\hline N01BB51 & bupivacaína $0,5 \%+$ glicose $-4 \mathrm{~mL}$ & 1 & 0 \\
\hline N01BB01 & bupivacaína isobárica $0,5 \%$ - $4 \mathrm{~mL}$ & 2 & 0 \\
\hline J01DB04 & cefazolina $1 \mathrm{~g}$ f/a & 3 & 0 \\
\hline N02BB02 & dipirona $500 \mathrm{mg} / \mathrm{mL}-2 \mathrm{~mL}$ & 5 & 5 \\
\hline R01AA03 & efedrina $50 \mathrm{mg} / \mathrm{mL}-1 \mathrm{~mL}$ & 5 & 3 \\
\hline $\mathrm{C} 01 \mathrm{CA} 24$ & epinefrina $1 \mathrm{mg} / \mathrm{mL}-1 \mathrm{~mL}$ & 5 & 0 \\
\hline C01CA01 & etilefrina $10 \mathrm{mg} / \mathrm{mL}-1 \mathrm{~mL}$ & 5 & 0 \\
\hline H02АB09 & hidrocortisona $500 \mathrm{mg}$ f/a & 1 & 0 \\
\hline N01BB10 & levobupivacaína isobárica $0,5 \%$ - $4 \mathrm{~mL}$ & 2 & 0 \\
\hline N01BB10 & levobupivacaína $0,5 \%$ - $20 \mathrm{~mL}$ & 1 & 0 \\
\hline S02DA01 & lidocaína $2 \%$ sem vasoconstritor - $5 \mathrm{~mL}$ & 5 & 5 \\
\hline D04AB01 & lidocaína geléia $30 \mathrm{~g}$ & 1 & 1 \\
\hline A03FA01 & metocloplamida $5 \mathrm{mg} / \mathrm{mL}$ - $2 \mathrm{~mL}$ & 2 & 0 \\
\hline N07AA01 & neostigmina $0,5 \mathrm{mg} / \mathrm{mL}-1 \mathrm{~mL}$ & 5 & 0 \\
\hline $\mathrm{A} 02 \mathrm{BC} 01$ & omeprazol $40 \mathrm{mg}$ f/a & 1 & 2 \\
\hline A4AA01 & ondasentrona $2 \mathrm{mg} / \mathrm{mL}$ - $2 \mathrm{~mL}$ & 2 & 2 \\
\hline N01BB09 & ropivacaína $1 \%$ - $20 \mathrm{~mL}$ & 1 & 2 \\
\hline N01AH01 & fentanil $0,5 \mathrm{mg} / \mathrm{mL}-5 \mathrm{~mL}$ & 1 & 0 \\
\hline N01AH01 & fentanil $0,5 \mathrm{mg} / \mathrm{mL}-2 \mathrm{~mL}$ & 0 & 2 \\
\hline N05CD08 & midazolan $5 \mathrm{mg} / \mathrm{mL}-3 \mathrm{~mL}$ & 1 & 1 \\
\hline V03AB25 & flumazenil $0,1 \mathrm{mg} / \mathrm{mL}$ & 0 & 2 \\
\hline $\mathrm{A} 01 \mathrm{AC} 02$ & dexametasona $4 \mathrm{mg} / \mathrm{mL}$ - 2,5 mL & 1 & 1 \\
\hline N01AX10 & propofol $1 \%-20 \mathrm{~mL}$ & 0 & 2 \\
\hline
\end{tabular}

Legenda: ATC - Anatomical Therapeutic Chemical - classificação segundo o ATC/DDD Index (www.whocc.no/atcddd). Fonte: Autores (2019).

Uma vez que a água destilada não tem seu consumo registrado em sistema, decidiu-se por manter a quantidade enviada atualmente deste item, que consiste em 9 ampolas. A atropina também teve sua quantidade atual mantida, já que se trata de um medicamento de emergência.

Para definição das quantidades de cada item, acrescentou-se o valor de duas vezes o desvio padrão à média, a fim de garantir a cobertura de maneira mais eficiente, pois, estatisticamente, tal recurso permite contemplar 95,46\% dos casos numa distribuição normal (Correa, 2003).

Nos casos de estimativas de quantidades em fração, os valores encontrados foram arredondados de acordo com as regras de arredondamento da $\mathrm{ABNT}$. E, nos casos de necessidade, estes ou outros medicamentos poderão ser solicitados à farmácia em quantidades adicionais. 


\subsection{Aspecto econômico}

Como quinta etapa do estudo se propôs uma comparação monetária entre o kit atual e o novo kit proposto.

A reformulação do kit atual tem como objetivo, além de melhores resultados acerca da segurança do paciente, reduzir o custo com uso de medicamentos nas microneurólises. A maior disponibilidade de medicamentos favorece o uso irracional, pois pode acarretar maior utilização do mesmo desnecessariamente, enquanto a redução desta disponibilidade tende a diminuir os números de utilizações desnecessárias. Também seria observada uma otimização no tempo de trabalho dos profissionais que têm dentre suas funções a montagem e a movimentação destes itens no estoque.

Conforme informações apesentadas na Figura 1, o kit atual é composto por 24 itens. Quando levada em consideração a quantidade de unidades, o número aumenta para 71. O valor agregado referente ao kit atual é de R\$217,14. Enquanto o novo kit proposto é composto por 14 itens (redução de 58,3\%), 48 unidades (32,3\% menos), custando R\$ 106,99, correspondendo a uma economia financeira de $\mathrm{R} \$ 110,15(50,7 \%)$ por procedimento.

Nas 76 cirurgias analisadas, nas quais se confirmou movimentação de medicamentos, a reformulação do kit geraria uma economia total de $\mathrm{R} \$ 8.371,40$, considerando-se o uso integral dos dois kits, o que equivale a uma economia mensal de aproximadamente $\mathrm{R} \$ 4.185,70$, estimativa que pode chegar a $\mathrm{R} \$ 5617,65$ por mês, se considerarmos os 102 kits que foram dispensados nos 2 meses em que se acompanharam virtualmente os procedimentos de microneurólise. Portanto, levando em consideração o grande volume de microneurólises realizadas, a adoção do novo kit poderia gerar impactos significativos financeiramente, qualitativa e quantitativamente em futuras estimativas de aquisição dos mesmos, bem como haveria redução no volume de documentações, movimentações, e espaço necessário para armazenamento.

\subsection{Aspectos gerais}

O acompanhamento cirúrgico feito presencialmente foi providencial para comprovar a suspeita de que a quantidade disponibilizada de medicamentos no kit atual excede consideravelmente a quantidade de medicamentos necessária para a realização do procedimento cirúrgico.

Durante a coleta de dados, algumas situações influenciaram diretamente na estimativa de utilização de medicamentos.

Por 26 vezes, os medicamentos utilizados não se encontravam relatados, não havendo movimentação de consumo de estoque de nenhum medicamento, causando subestimativas importantes.

Além da subestimativa, a falta de dados sobre a utilização de medicamentos implica em dificuldades importantes no que tange à segurança do paciente. Uma vez que não são registrados quando utilizados, em casos de danos ao paciente, há a inviabilidade de rastreamento dos possíveis causadores, impossibilitando a identificação de seus dados, tais como lote, validade e fornecedor.

Um kit contendo menos medicamentos, qualitativa e quantitativamente, acarreta a troca deste com maior frequência. A falta de excessos impede que este conjunto seja utilizado em mais de uma cirurgia. Assim, é possível que seja mitigada a falta de registro da utilização de medicamentos, resultando em benefícios às estratégias de segurança do paciente.

Os resultados indicam que algumas vezes se deixou de adotar os princípios da prática baseada em evidências. Como pode ser exemplificado pela utilização de antibioticoprofilaxia não justificada, visto que o procedimento em estudo é considerado como cirurgia limpa, não existem evidências científicas suficientes que comprovem o estabelecimento da eficácia deste tipo de farmacoterapia (Hospital Sírio-Libanês, 2015).

O custo médio com medicamentos por procedimento foi de $\mathrm{R} \$ 64,16$. Já o coeficiente de variação aplicado ao procedimento foi de $198 \%$, chegando ao valor de R\$ 1.078,89 em um procedimento. Através deste coeficiente de variação, é possível concluir que, apesar de ter um baixo custo, em média, a realização de algumas cirurgias obteve alto valor, quando se considerou o valor em medicamentos utilizados. 
Vale ressaltar que a cirurgia com o custo exorbitante foi realizada no centro cirúrgico voltado para cirurgias que se enquadram no regime de internação de longa duração. Tal centro cirúrgico normalmente abrange cirurgias mais complexas.

O procedimento em questão apresentou um perfil de utilização que diferia expressivamente das microneurólises observadas durante todo o estudo. É possível que os medicamentos registrados não correspondam somente à utilização do kit para uma microneurólise, mas sim à sua utilização por mais de uma vez, em uma mesma sala e que sua devolução só tenha sido realizada quando a reposição de medicamentos se mostrava imprescindível.

A observação de valores tão discrepantes em relação a um mesmo tipo de procedimento é motivo de alerta, apontando para o mau uso do dinheiro público. Caso o mesmo cenário acontecesse em um ambiente privado, onde os custos dos medicamentos fossem arcados por planos de saúde, estes medicamentos seriam glosados. Assim, os custos provavelmente seriam glosados e teriam que ser arcados pela própria instituição de saúde, gerando prejuízos.

Em relação ao número de itens utilizados, se obteve importante coeficiente de variação de $76 \%$. Em média, houve a utilização de 6,13 itens, podendo chegar a 17 itens, na cirurgia com o maior número de itens utilizados.

$\mathrm{Na}$ análise monetária, o coeficiente de variação é de 198\%. O custo médio do procedimento cirúrgico é de $\mathrm{R} \$ 64,16$. O procedimento com maior custo foi de $\mathrm{R} \$ 1.078,89$.

Os grandes valores de coeficiente de variação provavelmente indicam que o kit atual não tem sido utilizado da melhor maneira, ou não atende adequadamente à demanda. Além disso, como já mencionamos, a disponibilidade de maior número de itens favorece o uso irracional, resultando em prejuízos financeiros e à segurança do paciente.

\section{Conclusão}

Os resultados encontrados permitiram traçar o perfil de utilização de medicamentos em microneurólises no cenário de estudo, indicando que o kit básico atual é subutilizado e favorece o uso irracional de medicamentos, podendo ter impactos negativos financeiro e na segurança do paciente.

A análise do perfil de utilização de medicamentos em microneurólises no período de estudo, à luz das evidências técnicas e científicas, propiciou a proposta de um novo kit específico para o procedimento em questão, com reduções significativas no elenco, na quantidade de itens e no valor de cada kit proposto, podendo representar uma economia substancial à instituição tanto no custo direto quanto nos indiretos.

A ocorrência de fichas de solicitação preenchidas de forma incompleta ou incorreta pode ser considerada uma limitação deste estudo, pois pode levar a uma subestimativa do consumo de medicamento nas cirurgias no período de estudo. Esse problema também indica que, além da reestruturação do kit, é necessária a reformulação da rotina, associada ao treinamento da equipe, visando o correto preenchimento da folha de solicitação de medicamentos. Assim como, deve ser considerada a possibilidade de se adotarem estratégias para a promoção de atualizações técnicas quanto aos protocolos terapêuticos entre os prescritores na equipe de cirurgia.

Novos estudos poderão analisar os impactos da adoção do kit proposto ao longo do tempo, bem como poderão ser realizados estudos para analisar a viabilidade e propor kits específicos para outras cirurgias que são realizadas com maior frequência.

\section{Agradecimentos}

Ao Instituto Nacional de Traumatologia e Ortopedia (INTO); ao Programa de Pós-graduação Lato Sensu Residência em Farmácia Hospitalar; ao Ministério da Saúde (MS); à Universidade Federal Fluminense (UFF). 


\section{Referências}

Abreu, M. P. de, Vieira, J. L., Silva, I. F. da, Miziara, L. E. P. G., \& Fófano, R. (2006). Eficácia do ondansetron, metoclopramida, droperidol e dexametasona na prevenção de náusea e vômito após laparoscopia ginecológica em regime ambulatorial. Estudo comparativo. Revista Brasileira de Anestesiologia, 56(1).

Ácido tranexâmico. (2018) Eliza Yukie Saito. São Paulo: Blau Farmacêutica S.A. Bula de medicamento.

Adren: epinefrina. (2019). Renato Silva. Borges/Sabará: Hipolabor Farmacêutica Ltda. Bula de medicamento.

Aida, S., Baba, H., Yamakura, T., Taga, K., Fukuda, S., \& Shimoji, K. (1999). The Effectiveness of Preemptive Analgesia Varies According to the Type of Surgery: A Randomized, Double-Blind Study. Anesthesia \& Analgesia, 89(3), 711.

Atropion: sulfato de atropina monoidratada. (2019). Eliza Yukie Saito. São Paulo: Blau Farmacêutica S.A. Bula de medicamento.

Barron, R. P., Benoliel, R., Zeltser, R., Eliav, E., Nahlieli, O., \& Gracely, R. H. (2004). Effect of dexamethasone and dipyrone on lingual and inferior alveolar nerve hypersensitivity following third molar extractions: preliminary report. Journal of Orofacial Pain, 18(1), 62-68.

Bauer, H. C., Duarte, F. L., Horliana, A. C. R. T., Tortamano, I. P., Perez, F. E. G., Simone, J. L., \& Jorge, W. A. (2013). Assessment of preemptive analgesia with ibuprofen coadministered or not with dexamethasone in third molar surgery: a randomized double-blind controlled clinical trial. Oral and Maxillofacial Surgery, 17(3), 165-171

Benseñor, F. E. M., \& Cicarelli, D. D. (2003). Sedação e analgesia em terapia intensiva. Revista Brasileira de Anestesiologia, 53(5), 680-693.

Bicarbonato de sódio 8,4\%. (2018) Irineu Marcellini. Ribeirão das Neves: Hypofarma-Instituto de Hypodermia e Farmácia Ltda.. Bula de medicamento.

Braga, E. L. C. (2017). Palonosetrona e ondansetrona na profilaxia de náuseas e vômitos pós-operatórios em mulheres com 60 anos ou mais submetidas a colecistectomias videolaparoscópicas: estudo aleatório e duplamente encoberto..Dissertação de mestrado.Universidade Federal Fluminense, Niterói, Rio de Janeiro.

Brasil. (2009) Agência Nacional De Vigilância Sanitária. Guia Regulatório - ANVISA: Glossário. Brasília, DF. Recuperado em 30 março de 2019 de http://portal.anvisa.gov.br/documents/33868/2894051/Gloss\%C3\%A1rio+da+Resolu\%C3\%A7\%C3\%A3o+RDC+n\%C2\%BA+4\%2C+de+10+de+fevereiro+d e+2009/61110af5-1749-47b4-9d81-ea5c6c1f322a

Bricola, S. A. P. de C., Santos, V. G., Toma, W., \& Martins, M. de A. (2018). Medicamentos: terapêutica segura. In observatorio.fm.usp.br. Manole.

Bridion: sugamadex. (2018). Grenville: Patheon Manufacturing Services LLC. Bula de medicamento.

Clonidina. (2019). São Paulo: Cristália Produtos Químicos Farmacêuticos Ltda.. Bula de medicamento.

Cloridrato de ranitidina. (2019). Ronoel Caza de Dio. Hortolândia: EMS S/A. Bula de medicamento.

CNPq. (2021). Glossário - Plataforma Lattes - CNPq. Glossário.

http://lattes.cnpq.br/web/dgp/glossario?p_p_id=54_INSTANCE_QoMcDQ9EVoSc\&_54_INSTANCE_QoMcDQ9EVoSc_struts_action=\%2Fwiki_display\%2

Fview\&_54_INSTANCE_QoMcDQ9EVoSc_nodeName=Main\&_54_INSTANCE_QoMcDQ9EVoSc_title=Pesquisa

Correa, S. M. B. B. (2003). Probabilidade e estatística

DEJEAN, K. S., SANTOS, I. R. M. dos, ANDRADE, F. V., \& SOUZA, L. M. de A. (2008). Analgesia preemptiva em odontologia. Publicatio UEPG Ciencias Biologicas E Da Saude, 14(2), 23-30.

Do Carmo, G.A. L., \& Caramelli, B. (2012) Avaliação perioperatória do paciente hipertenso. Rev. bras. hipertens, 19(1), 3-10.

Efedrin: sulfato de efedrina. (2018). José Carlos Módolo. São Paulo: CRISTÁLIA Produtos Químicos Farmacêuticos Ltda.. Bula de medicamento.

Etilefril: cloridrato de etilefrina. (2018). Pouso Alegre: União Química Farmacêutica Nacional S/A. Bula de medicamento.

Fentanyl Interactions Checker. (n.d.). Drugs.com. Recuperdo em 28 de novembro de 2019 de https://www.drugs.com/interactions-check.php?drug_list=1074-0

Flumazenil. (2019). Pouso Alegre: União Química Farmacêutica Nacional S/A. Bula de medicamento.

Garcia, J. B. S., Issy, A. M., \& Sakata, R. K. (2001). Analgesia preemptiva. Revista Brasileira de Anestesiologia, 51(5), 448-463.

Hollander, J. L. (1970). Intrasynovial corticosteroid therapy in arthritis. Maryland State Medical Journal, 19(3), 62-66.

Hospital Sírio-Libanês. (2015). Protocolo de antibioticoprofilaxia no paciente cirúrgico. Recuperado em 22 de outubro de 2019 em https://www.hospitalsiriolibanes.org.br/institucional/gestao-da-qualidade/Documents/2018-11-01-protocolos/Protocolo\%20Antibioticoprofilaxia\%

20no\%20Paciente\%20Cir\%C3\%BArgico\%20e\%20do\%20Centro\%20Diagn\%C3\%B3stico/Manual\%20Antibioticoprofilaxia-paciente-cirurgico_150924.pdf

Ketamin: dextrocetamina. (2018). José Carlos Módolo.Itapira: CRISTÁLIA Produtos Químicos Farmacêuticos Ltda.. Bula de medicamento.

Kohn, L. T., Corrigan, J., Donaldson, M. S., \& America, I. (1999). To err is human: building a safer health system. National Academy Press, , Cop.

Lei $\mathrm{n}^{\circ} 11.903$, de 14 de janeiro (2009). Dispõe sobre o rastreamento da produção e do consumo de medicamentos por meio de tecnologia de captura, armazenamento e transmissão eletrônica de dados. Brasília, DF. Recuperado em 30 de março de 2019 de http://www.planalto.gov.br/ccivil_03/_Ato20072010/2009/Lei/L11903.htm 
Research, Society and Development, v. 10, n. 14, e486101422376, 2021

(CC BY 4.0) | ISSN 2525-3409 | DOI: http://dx.doi.org/10.33448/rsd-v10i14.22376

Lei $\mathrm{n}^{\circ}$ 13.410, de 28 de dezembro (2016). Altera a Lei no 11.903, de 14 de janeiro de 2009, para dispor sobre o Sistema Nacional de Controle de Medicamentos. Brasília, DF. Recuperado em 29 de abril de 2019 de http://www.planalto.gov.br/ccivil_03/_ato2015-2018/2016/lei/L13410.htm

Metilsulfato de neostigmina. (2018). Eliza Yukie Saito. São Paulo: Blau Farmacêutica S.A,. Bula de medicamento.

Míriam Elias Cavallini, \& Marcelo Polacow Bisson. (2002). Farmácia hospitalar: um enfoque em sistemas de saúde. Manole.

Neocaína pesada: cloridrato de bupivacaína + glicose 8\%. (2018). José Carlos Módolo. Itapira: Cristália Produtos Químicos Farmacêuticos Ltda,. Bula de medicamento.

Nociti, J. R. (2017). Ropivacaine: the newest anesthetic agent celebrates 20 years. Revista Dor, 18(4).

Novabupi: Cloridrato de Levobupivacaína. (2018). José Carlos Módolo. Itapira: Cristália Produtos Químicos Farmacêuticos Ltda. Bula de medicamento.

Omeprazol sódico. (2019). José Carlos Módolo.Itapira: Cristália Produtos Químicos Farmacêuticos Ltda. Bula de medicamento.

PARANÁ. (2001) Secretaria de Saúde. Código Sanitário do Paraná. Curitiba, PR. Recuperado em 30 de março de 2019 de <http://www.saude.pr.gov.br/modules/conteudo/conteudo.php?conteudo=665>

Pereira, C. U., Carvalho, A. F. de, Carvalho, M. F. de, \& Nascimento, T. F. do. (2005). Tratamento conservador da síndrome do túnel do carpo: revisão da literatura. Ri.ufs.br.

Provive: propofol. (2018). Índia: Claris Produtos Farmacêuticos do Brasil Ltda. Bula de medicamento.

Righeti, C. C. F., \& Lauretti, G. R. (2014). Avaliação do efeito analgésico da dexametasona administrada por via peridural na dor aguda pós-operatória de pacientes submetidos à cirurgias ortopédicas do joelho. Universidade de São Paulo, Ribeirão Preto.

Succinil colin: cloreto de suxametônio. (2018). Florentino de Jesus Krencas. Embu-Guaçu: União Química Farmacêutica Nacional S/A. Bula de medicamento.

Vicente, Â., Loffi, A. O. B., \& Nesi, H. (2013). Uso de corticosteroide no pré-operatório em cirurgia de terceiros molares. Revista Brasileira de Odontologia, 70(1), 22-27. 